\title{
En Bloc Resection of Neuroendocrine Tumor of Colon: Case Report and Review of the Literature
}

\author{
Ana Lilia Pacheco-Carrillo ${ }^{1}$, José Antonio Ortega-Jiménez ${ }^{1 *}$, Mirza Palacios-Rojo ${ }^{2}$, Roberto \\ Ángel Núñez-González ${ }^{1}$, Aldo Joaquín Díaz-Aguilar ${ }^{1}$, Antonio Silvestre Sosa-Luis ${ }^{1}$, José Galaor \\ Gutiérrez-Durante $^{1}$, Carlos Arturo Córdova-Bravo ${ }^{1}$, José Eduardo Zúñiga-Vázquez ${ }^{1}$ \\ ${ }^{l}$ Department of General Surgery, Mexican Institute of Social Security, High Specialty Medical Unit of Puebla, \\ Puebla, Mexico \\ ${ }^{2}$ Department of Internal Medicine, Health Services of Veracruz, Regional Hospital of Rio Blanco, Veracruz, \\ Mexico \\ *Corresponding Author: José Antonio Ortega-Jiménez, Department of General Surgery, Mexican \\ Institute of Social Security, High Specialty Medical Unit of Puebla, Puebla, Mexico.
}

\begin{abstract}
Neuroendocrine tumors (NETS) of the colon are rare and aggressive neoplasms that account for $<1 \%$ of all colorectal cancer. The estimated incidence of NETs of the colon and rectum is 0.3-1.1 per 100,000 in developed nations. The patients present vague and non-specific symptoms, including abdominal pain, weight loss, bleeding, obstruction, and constipation, causing a delay in the diagnosis. Imaging studies are necessary for de study protocol; computed tomography is often the initial imaging study, useful for disease staging and surgical planning. Surgery is the only curative treatment for NETs and recommended for most patients whose imaging studies suggest that complete resection is possible. We present the case of a 49-year-old male with a neuroendocrine tumor of the sigmoid colon with the involvement of surrounding organs, successfully treated by en bloc resection.
\end{abstract}

Keywords: Neuroendocrine Tumor of Colon, En Block Resection, Colorectal Surgery

\section{INTRODUCTION}

Neuroendocrine carcinoma of the large intestine and rectum is an uncommon type of neuroendocrine tumor (NET) that accounts for $<1 \%$ of all colorectal cancer [1].The incidence of NETs of the colon and rectum has been increasing in the past decades, thought due in part to increased screening colonoscopies. Although still uncommon, with an estimated incidence of 0.3 and 1.1 per 100,000 in developed nations [2]. The clinical development of NET of the colon includes very destructive and aggressive growth, followed by rapid spread [1] and usually presents with non-specific symptoms, most commonly bleeding, abdominal pain, change in bowel habits, anorexia, weight loss, and weakness [3]. These non-specific manifestations are frequently misdiagnosed, causing a delay in diagnosis and decreasing the chances to perform a successful surgical treatment.

\section{CASe Report}

We present the case of a 49-year-old male with a 2-year history of intermittent abdominal pain on the left lower quadrant who presented to the emergency room with hematochezia. The patient also referred unquantified weight loss and change in bowel habits (constipation) for the last four months. He had received multiples previous medical examinations, and he was symptomatically treated with no relief. In his medical history, he denied diseases or previous surgical procedures. During the interrogation in the emergency room, he reported severe abdominal pain on the left lower quadrant and hematochezia of 12 hours. The patient was reported with normal vital signs, and at physical examination, he looked pale and dehydrated; an abdominal tumor was palpable on the left lower quadrant, and the rectal exam showed blood remains. The laboratory data on admission showed hemoglobin $(6.5 \mathrm{~g} / \mathrm{dL})$, leukocytes $(11,700 / \mathrm{mm} 3)$, platelets $(546,000 / \mathrm{L})$, creatinine 
(1.5 mg/dL), glucose (137 $\mathrm{mg} / \mathrm{dL})$, carcinoembryonic antigen $(9.53 \mathrm{ng} / \mathrm{ml})$ and normal clotting times. The patient was treated with symptomatic management and with blood transfusion, reaching normal levels of hemoglobin. An abdominal and pelvic computed tomography revealed a bulky pelvic tumor involving large and small intestine [Figure 1]. Colonoscopy was performed, finding a lesion at $50 \mathrm{~cm}$ of the anal margin that did not allow the instrument to pass. Colo TAC was also made, showing a large tumor of the sigmoid colon [Figure 2]. Later the patient presented severe generalized abdominal pain, distension, inability to pass gas or stool, and vomiting. He received conservative management for bowel obstruction. However, he did not show improvement, so we decided to perform a laparotomy.

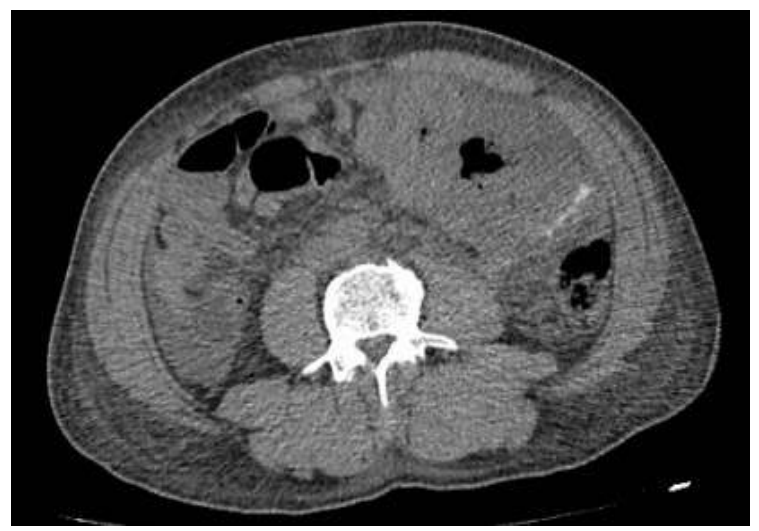

Figure1. Abdominal and pelvic computed tomography, axial plane, demonstrating a bulky pelvic tumor involving the large and small intestine

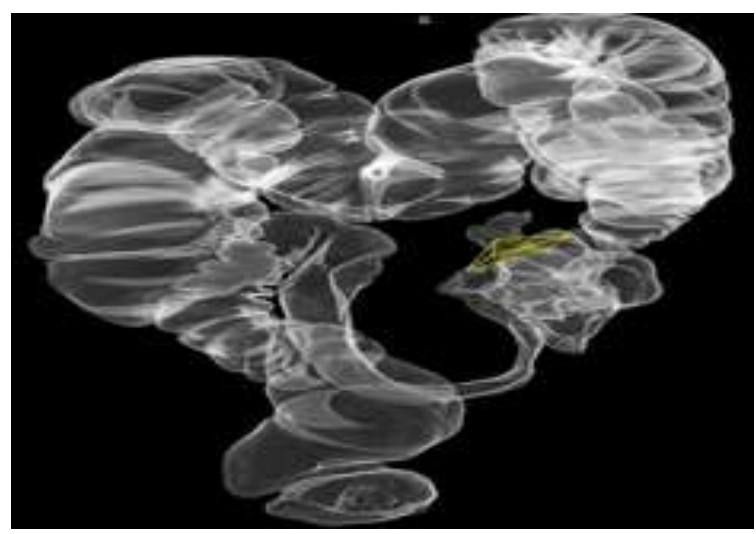

Figure2. Reconstructed 3D images of ColoTAC showing voluminous tumors in the sigmoid colon

During the surgery, we found a sigmoidal tumor of $20 \times 15 \mathrm{~cm}$ occluding the $90-95 \%$ of the lumen [Figure 3], invading rectus muscles, small intestine (ileum) and adhered to the bladder dome but without infiltration.

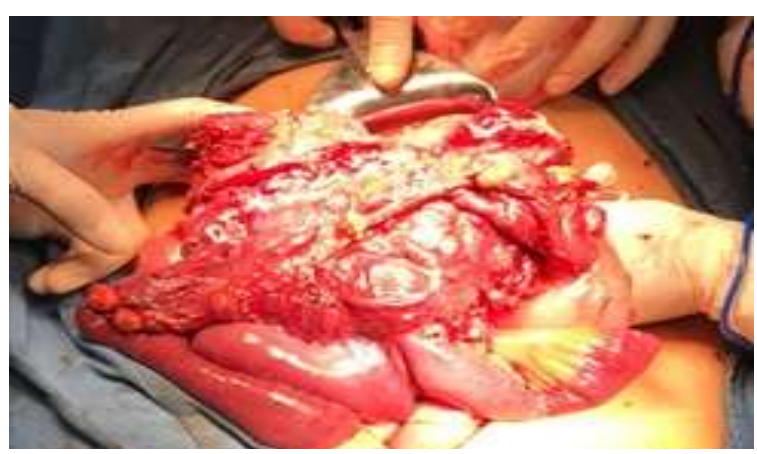

Figure3. Bulky tumor in sigmoid colon with irregular borders and involvement of adjacent structures

We made total resection of the sigmoidal colon, providing tumor-free margins of $10 \mathrm{~cm}$, partial resection of rectus muscles, resection of the bladder dome, resection of $20 \mathrm{~cm}$ of ileum [Figure 4], primary colorectal anastomosis, primary repair of the bladder, primary anastomosis of the small intestine and repair of the abdominal wall. The histopathology of the surgical piece [Figure 5] reported neuroendocrine carcinoma of $14 \mathrm{~cm}$, with areas of necrosis, CK positive, CK20 positive, synaptophysin positive [Figure 6], K167 positive, chromogranin negative, CK7 negative, and surgical margins without tumor. The patient presented good evolution, and he was discharged home nine days after the surgery.

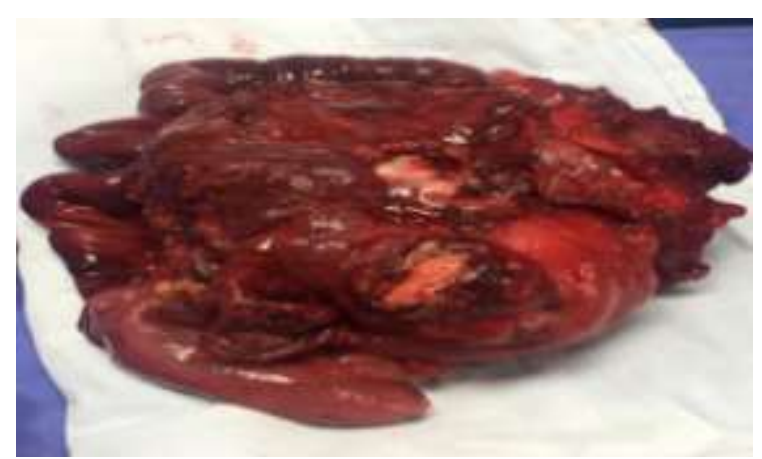

Figure4. Surgical piece after en bloc resection of the entire tumor of the sigmoid colon

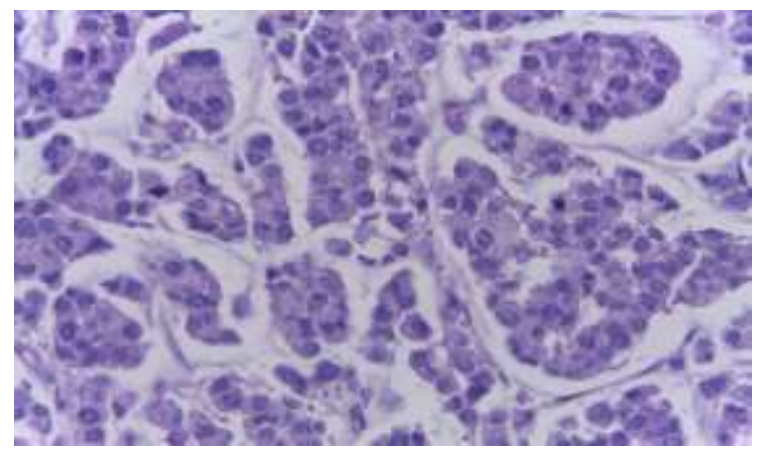

Figure5. Photomicrograph of the surgical piece reported malignant neoplasm with a growth pattern of nests and cords, round nuclei, and abnormal mitoses 


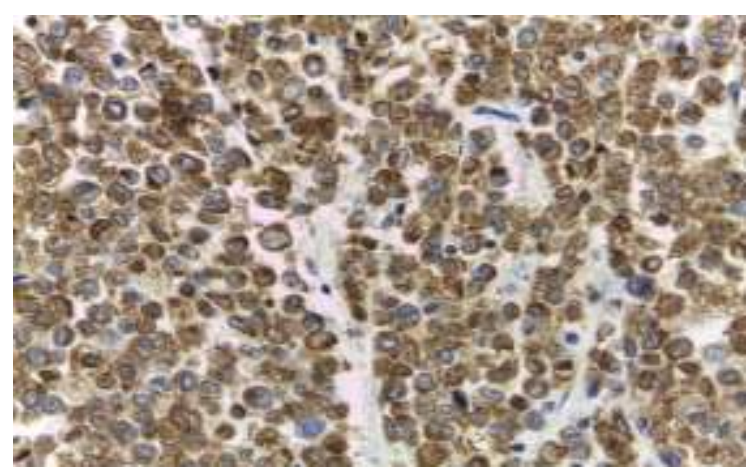

Figure6. Photomicrograph of immunostained sections revealed tumor cells showed a positive reaction for synaptophysin

\section{DISCUSSION}

Approximately $7.5 \%$ of NETs have a colonic origin. NETs of the colon and rectum are often discovered incidentally during screening colonoscopies. Perhaps related to improved colon cancer screening, colon NETs have increased over the last three decades. The median age at diagnosis is 65 years [4]. Neuroendocrine carcinoma is known for its overly aggressive progression, and its pathogenesis is still not understood [5], characterized by an organoid and diffuse growth of highly atypical, small to large, neoplastic cells, with more than 20 mitotic figures per 10 high-power fields $(\times 400)$, a Ki-67 proliferative index greater than $20 \%$ and large areas of geographic necrosis [6]. These aggressive tumors cause serious health problems, such as colonic obstruction and exclusion of internal organs; clinically, it results in a poor prognosis [1]. They are often larger than $2 \mathrm{~cm}$, and approximately two-thirds of the cases have either regional or distant metastasis at the time of diagnosis [2].

Colonic NETs are more likely to present with symptoms that tend to be vague and nonspecific, and more often, these symptoms are related to the mass effects of the tumor. These can include vague abdominal pain, weight loss, bleeding, obstruction, and constipation, although most patients have no symptoms [3].

Computed tomography (CT) is the initial imaging study for a patient presenting signs or symptoms of a suggestive NET. These studies are most useful for disease staging and surgical planning as they provide excellent anatomic detail of the tumors themselves and surrounding structures. In primary NETs, the average sensitivity of a CT scan is $73 \%$. This modality is also useful when the primary tumor site is unknown [7]. The role of the tomography, in this case, was essential to submit the patient to surgery.

Surgery is the only curative treatment for NETs and is recommended for most patients for whom cross-sectional imaging suggests that complete resection is possible. Although NETs generally have a better prognosis than adenocarcinomas at the same site [8]. For carcinoid tumors $>2 \mathrm{~cm}$, a standard cancer resection as performed for adenocarcinomas is mandatory [9]. Due to the size and characteristics of the tumor, the complete resection was performed, including adjacent structures.

The overall 5-year survival rates reported for colon carcinoids are significantly better than those for adenocarcinomas, with an average of $37 \%$ [9]. With the surgical treatment performed in this patient, an improvement in the life expectancy is expected.

\section{Conclusions}

Colonic NETs are aggressive neoplasms. They present with non-specific symptoms, causing a delay in the diagnosis. The patients' survival will depend primarily on the possibility of performing a complete resection of the tumor, so a multidisciplinary team of experts in the field should treat them.

\section{REFERENCES}

[1] Warsinggih, Liliyanto, Prihantono, Ariani GDW, Faruk M. Colorectal neuroendocrine tumors: A case series. Int J Surg Case Rep. 2020;72:411-7.

[2] Ford MM. Neuroendocrine Tumors of the Colon and Rectum. Dis Colon Rectum. 2017;60 (10):1018-20.

[3] Eggenberger JC. Carcinoid and other neuroendocrine tumors of the colon and rectum. Clin Colon Rectal Surg. 2011;24 (3):129-34.

[4] Hrabe J. Neuroendocrine Tumors of the Appendix, Colon, and Rectum. Surg Oncol Clin N Am. 2020;29 (2):267-79.

[5] Onoda N, Kobayashi H, Satake K, Ishikawa T, Maeda K, Chung K H, et al. Neuroendocrine carcinoma of the sigmoid colon: report of a case. Surg Today. 1999;29 (10):1079-82.

[6] Bolzacchini E, Chini C, Cortelezzi CC, Vallini I, Pinotti G, La Rosa S, et al. Poorly Differentiated Neuroendocrine Carcinoma of the Sigmoid Tract in Long-Standing Ulcerative Colitis: Report of a Case and Review of the Literature. Int J Surg Pathol. 2018;26 (5):479483. 
[7] Maxwell JE, Howe JR. Imaging in neuroendocrine tumors: an update for the clinician. Int J Endocr Oncol. 2015;2 (2):15968.

[8] Yao JC, Hassan M, Phan A, Dagohoy C, Leary $\mathrm{C}$, Mares JE, et al. One hundred years after "carcinoid": epidemiology of and prognostic factors for neuroendocrine tumors in 35,825 cases in the United States. J Clin Oncol. 2008;26 (18):3063-72.

[9] Rothmund M, Kisker O. Surgical treatment of carcinoid tumors of the small bowel, appendix, colon, and rectum. Digestion. 1994;55 (3):86-9.

Citation: Ana Lilia Pacheco-Carrillo, José Antonio Ortega-Jiménez, Mirza Palacios-Rojo, Roberto Ángel Núñez-González, Aldo Joaquín Díaz-Aguilar, Antonio Silvestre Sosa-Luis, José Galaor Gutiérrez-Durante, Carlos Arturo Córdova-Bravo, José Eduardo Zúñiga-Vázquez. "En Bloc Resection of Neuroendocrine Tumor of Colon: Case Report and Review of the Literature”. ARC Journal of Surgery. 2020; 6(2):14-17. DOI:https://doi.org/ 10.20431/2455-572X.0602003.

Copyright: (C) 2020 Authors. This is an open-access article distributed under the terms of the Creative Commons Attribution License, which permits unrestricted use, distribution, and reproduction in any medium, provided the original author and source are credited. 